\title{
Correspondence
}

Korean J Ophthalmol 2021:35(1):89-90 https://doi.org/10.3341/kjo.2020.0094

\section{Formation of Full Thickness Macular Hole Following Pars Plana Vitrecto- my and Internal Limiting Membrane Peeling and Its Spontaneous Closure}

\section{Dear Editor,}

A macular hole is a common ophthalmic condition which results in reduced vision. It is widely accepted that it is treated by vitrectomy and internal limiting membrane (ILM) peel, although spontaneous closure is not uncommon. Here we report a case where an epiretinal membrane and a lamellar hole were treated by vitrectomy and ILM peel. It resulted in a full thickness macular hole (FTMH) soon after the operation which then spontaneously closed.

An 80-year-old female presented to the clinic with reduced vision in the right eye in the last 2 years. She did not have any past ophthalmic history. Vision was $6 / 15$ - 2 in the right eye (RE) and 6 / 7.5 - 1 in the left eye in Snellen chart. On examination, the anterior segments were normal with intraocular pressures of $10 \mathrm{mmHg}$ in both eyes. There was bilateral nuclear sclerosis. Fundus examination and optical coherence tomography (OCT) of the right macula showed lamellar hole and epiretinal membrane (Fig. 1A). She underwent uncomplicated phacoemulsification, intraocular lens insertion, pars plana vitrectomy, ILM peel of the macula within the vascular arcade and air in the RE.

She was seen 14 days postoperatively in clinic and the visual acuity (VA) RE was 6 / 38 improving to 6 / 9.5 - 2 with pinhole. On macula OCT, the configuration of the fovea was better compared to preoperative scan (Fig. 1B). She was followed up again in 6 weeks and VA was $6 / 38$

Received: July 18, 2020 Final revision: September 3, 2020 Accepted: September 14, 2020 improving to 6 / 24 - 1 with pinhole. On the macula OCT, it was noted that the fovea dip was slightly deeper compared to the last scan (Fig. 1C). In the next visit, which was 14 weeks postoperative, VA RE 6 / 18 - 1 with refraction and it was noted that a FTMH had formed (Fig. 1D). Following discussion of the options with the patient, we have listed her for surgical closure of the macular hole. However, when she was seen 4 weeks later, the hole was almost closed (Fig. 1E). VA was $6 / 15+2$ with glasses. Four weeks following this visit, VA was still $6 / 15+2$ and the scan was stable with minimal retinal outer layer disruption with closed hole (Fig. 1F).

Formation of FTMH following vitrectomy is rare with its incidence being reported as $0.24 \%$ [1]. It has been shown in the literature that such FTMHs can close spontaneously in many different clinical scenarios [2]. Distelmaier et al. [3] reported that a FTMH can recur following surgical repair but it was closed without any intervention. Sponta-

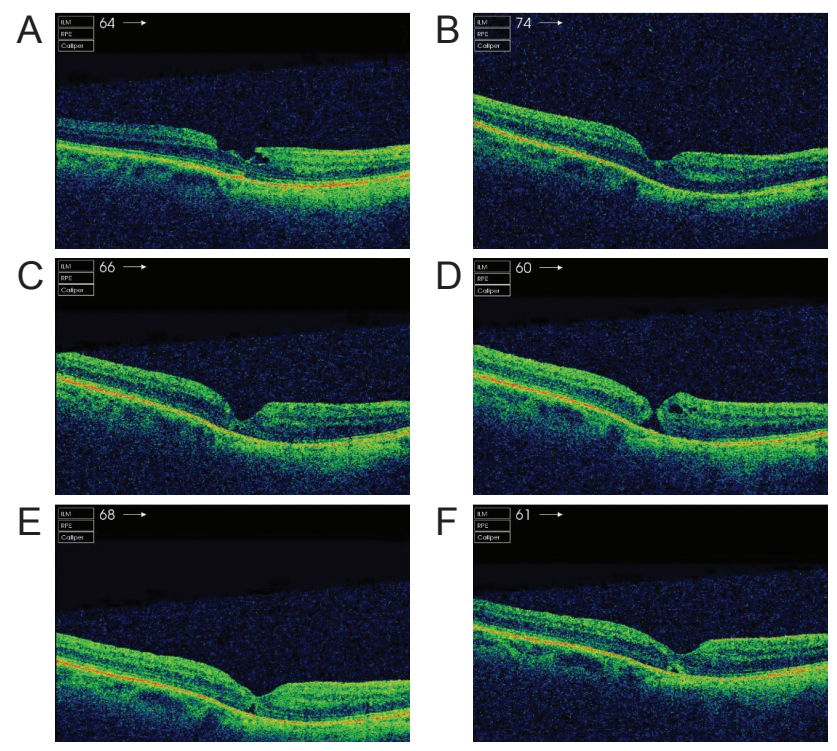

Fig. 1. (A) Preoperative macular optical coherence tomography of the right eye. Macula optical coherence tomography of the right eye (B) 14 days, (C) 8 weeks, (D) 14 weeks, (E) 18 weeks, and (F) 22 weeks postoperative. 
neous closure has also been reported in context of posterior uveitis, cystoid macular oedema [4], and following retinal detachment repair by pars plana vitrectomy [5]. It is possible that the ILM peel disrupted the foveal surface which triggered the formation of FTMH, but at the same time promoting closure by removing the traction from the vitreous. Lee et al. [1] discussed in their study the two mechanisms of secondary macular hole formation following vitrectomy. One mechanism is particularly relevant in our case where observed that small cysts were present in the retinal layers which merged to form a larger cyst. It can either rupture or dehisce, leading to a FTMH by degenerative process. In the macula OCT (Fig. 1D), there is evidence of intraretinal cyst and this may have played a major role in FTMH.

We report the first case where a FTMH was seen for the first-time following vitrectomy and ILM peel which then spontaneously closed. Epiretinal membrane is a common condition which results in significant symptoms such as distortion and blurred vision. Surgical intervention is frequently employed to improve vision but complications such as a FTMH would result in worse vision postoperatively and patient dissatisfaction. Our case demonstrates that in the event of such a complication, it may be reasonable to observe before considering second surgery, which is accompanied by further risks.
Christina S Lim, Abdul El-Khayat, Aashish Mokashi

Department of Ophthalmology, University Hospitals of

Leicester NHS Trust, Leicester, United Kingdom

E-mail (Christina S Lim): christina.sk.lim@doctors.org.uk

\section{Conflict of Interest}

No potential conflict of interest relevant to this article was reported.

\section{References}

1. Lee SH, Park KH, Kim JH, et al. Secondary macular hole formation after vitrectomy. Retina 2010;30:1072-7.

2. Sheng Y, Sun W, Shen Y. Delayed closure of macular hole secondary to Terson syndrome after vitrectomy: a case report and literature review. Medicine (Baltimore) 2019;98:e16577.

3. Distelmaier P, Meyer LM, Fischer MT, et al. Delayed macular hole closure. Case Rep Ophthalmol 2014;5:121-4.

4. Elhusseiny AM, Smiddy WE, Flynn HW, Schwartz SG. Case series of recurring spontaneous closure of macular hole. Case Rep Ophthalmol Med 2019;2019:2398342.

5. Luo W, Song W, Jiang L, Zhu Y. Rhegmatogenous retinal detachment due to full-thickness macular hole secondary to uveitis: a case report. Int J Clin Exp Med 2018;11:1277881. 